\title{
Thymalin: Activation of Differentiation of Human Hematopoietic Stem Cells
}

\author{
V. Kh. Khavinson ${ }^{1,2}$, N. S. Linkova ${ }^{1,3}$, I. M. Kvetnoy ${ }^{1}$, V. O. Polyakova ${ }^{1}$, \\ A. O. Drobintseva ${ }^{1}$, T. V. Kvetnaia ${ }^{1}$, and O. M. Ivko ${ }^{1}$
}

Translated from Kletochnye Tekhnologii v Biologii i Meditsine, No. 3, pp. 158-163, September, 2020

Original article submitted June 26, 2020

\begin{abstract}
Thymalin is a polypeptide complex isolated from the thymus and regulating the functions of the immune system. Thymalin is effective in therapy of acute respiratory syndrome, chronic obstructive bronchitis, and other immunopathology. Thymalin increases functional activity of $\mathrm{T}$ lymphocytes, but the targeted molecular mechanism of its biological activity requires further study. We studied the influence of thymalin on differentiation of human hematopoietic stem cells (HSC) and expression of CD28 molecule involved in the implementation of antiviral immunity in COVID-19 infection. It was found that thymalin reduced the expression of CD44 (stem cell marker) and CD117 (molecule of the intermediate stage of HSC differentiation) by 2-3 times and increased the expression of CD28 (marker of mature T lymphocytes) by 6.8 times. This indirectly indicates that thymalin stimulated differentiation of $\mathrm{CD} 117^{+}$cells into mature CD28 $8^{+} \mathrm{T}$ lymphocytes. It is known that in patients with severe COVID-19, the number of $\mathrm{CD} 28^{+}, \mathrm{CD}^{+}, \mathrm{CD} 8^{+} \mathrm{T}$ lymphocytes in the blood decreased, which attested to a pronounced suppression of immunity. It is possible that the antiviral effect of thymalin consists in compensatory stimulation of HSC differentiation into CD28 $8^{+} \mathrm{T}$ lymphocytes at the stage of immunity suppression in unfavorable course of viral infection. Thymalin can be considered as an immunoprotective peptide drug for the prevention of COVID-19.
\end{abstract}

Key Words: thymalin; peptides; hematopoietic stem cells; cell differentiation; COVID-19

The search for effective and safe antiviral agents has now become a priority task in molecular biology and medicine. In March 2020, the World Health Organization recognized COVID-19 (a disease caused by the SARS-CoV-2 virus, severe acute respiratory syndrome coronavirus 2) as a pandemic. Currently, there are no effective specific treatments for COVID-19. One of the pathogenetic factors in the development of COVID-19 is activation of the synthesis of proinflammatory cytokines by lung macrophages, which in severe cases

\footnotetext{
${ }^{1}$ Department of Biogerontology, St. Petersburg Institute of Bioregulation and Gerontology, St. Petersburg, Russia; ${ }^{2}$ Group of Peptide Regulation and Aging, I. P. Pavlov Institute of Physiology, Russian Academy of Sciences, St. Petersburg, Russia; ${ }^{3}$ Department of Therapy, Geriatrics, and Anti-Aging Medicine, Academy of Postgraduate Education under Federal Research Clinical Center of Federal MedicalBiological Agency of Russia, Moscow, Russia. Address for correspondence: linkova8101984@gmail.com. N. S. Linkova
}

leads to the development of an inflammatory reaction and distress syndrome. In severe form of COVID-19, the immune response is overactivated; it leads to a cytokine storm, which can be fatal [10].

Thymalin is a complex of peptides with a molecular weight of up to $10 \mathrm{kDa}$ isolated from cattle thymus [6]. Thymalin normalizes cellular and humoral immunity and functions of the hemostasis system, exhibits antioxidant properties, stimulates phagocytosis, regeneration, and hematopoiesis in case of their suppression, and normalizes the function of $\mathrm{T}$ lymphocytes. Thymalin also acts as a geroprotector; it prolongs animal lifespan and decreases the incidence of tumors $[1,6,15]$. The use of thymalin in infectious diseases (acute and chronic hepatitis A and B, meningitis, typhoid fever, dysentery, pseudotuberculosis, acute respiratory viral infection, and influenza), as well as in acute pneumonia, chronic obstructive bronchitis, lung 
abscess, and bronchial asthma is accompanied by normalization of the immunogram, including a decrease in the level of proinflammatory cytokines [7]. It should be emphasized that no adverse or allergic reactions were noted during many years of experimental study and clinical use of thymalin. The active components of thymalin are di- and tripeptides that regulate gene expression and synthesis of proteins of differentiation, proliferation, and apoptosis of immune cells [5]. The molecular mechanism of the effect of thymalin on stem cell differentiation and its antiviral effect requires further study.

Human hematopoietic stem cells (HSC) originate from hemangioblasts and are located in the red bone marrow. HSC are called pluripotent because they give rise to myeloid and lymphoid blood cells. Myeloid cells differentiating from HSC include monocytes, macrophages, granulocytes (neutrophils, basophils, and eosinophils), erythrocytes, megakaryocytes, platelets, and myeloid dendritic cells. The lymphoid cell line, which is derived from HSC, includes $\mathrm{T}$ and $\mathrm{B}$ lymphocytes, NK cells, and lymphoid dendritic cells. Thus, HSC are an important reserve for differentiation and maintenance of the pool of functionally active immune cells.

Our aim was to study the effect of thymalin on differentiation of human stem cells and expression of the CD28 molecule involved in the implementation of antiviral immunity in COVID-19.

\section{MATERIALS AND METHODS}

HSC were isolated from umbilical cord blood using a protocol for isolation of mononuclear cells developed by GE Healthcare Company. The blood was obtained during separation of the placenta during physiological childbirth in the maternity ward of the D. O. Ott Research Institute of Obstetrics, Gynecology, and Reproductology (St. Petersburg). The blood was diluted 1:1 with Dulbecco's PBS (DPBS) and transferred to a tubed with $3 \mathrm{ml}$ Ficoll-Paque Plus (GE Healthcare). After centrifugation at $400 \mathrm{rpm}$ for $30 \mathrm{~min}$, the upper layer of plasma was discarded and the layer containing mononuclear cells was carefully collected and transferred to another tube, mixed 1:3 with DPBS, and centrifuged at $400 \mathrm{rpm}$ for $10 \mathrm{~min}$. The supernatant was discarded, the cells were suspended in $5 \mathrm{ml}$ RPMI-1640 culture medium, transferred to a vial with a non-adhesive surface, and incubated in a thermostat at $5 \%$ and $37^{\circ} \mathrm{C}$. All HSC cultures were divided into 2 groups: control (addition of nutrient medium) and experimental addition of thymalin in a concentration of $100 \mathrm{ng} / \mathrm{ml}$ (batch 70512, Samson-Med). The cells were cultured up to passages 2 and 7; thymalin was added to the HSC cultures during each passage.
The expression of signaling molecules was verified by immunofluorescence using primary monoclonal antibodies to CD44 (1:50, Dako), CD28 (1:50, Novocastra), and CD117 (1:150, Abcam). The cells were fixed with $4 \%$ paraformaldehyde for $10 \mathrm{~min}$. HSC were incubated with $0.5 \%$ Triton X-100 for 10 min at room temperature. The cells were incubated with Protein Block (Dako) for 10 min to block nonspecific binding sites. HSC were incubated with primary antibodies for $60 \mathrm{~min}$ in a thermostat at $37^{\circ} \mathrm{C}$, and then with secondary antibodies conjugated with Alexa Fluor 488 or 647 (1:1000, Abcam) for $30 \mathrm{~min}$ at room temperature in the dark. The nuclei were counterstained with DAPI (Sigma). Finally, the preparations were embedded in a Dako Fluorescent Mounting Medium (Dako) under coverslips.

The micropreparations were examined under a confocal laser scanning microscope FV 1000 (Olympus) at $\times 200$ using FW10 software to obtain micrographs with the results of immunocytochemical studies ( 5 fields of view in each sample). The micrographs were analyzed using ImageJ software by two parameters: relative expression area (\%) and mean brightness (arb. units). The relative area of expression of the marker was calculated as the ratio of the area of immunopositive cells to the total area of the preparation. The relative area of the expression is characterized by the number of immunostained cells. The mean brightness reflects marker concentration in one cell.

The results were statistically processed using the Statistica 10.0 software (StatSoft, Inc.). Comparison of the mean values of studied parameter in groups 1 and 2 was carried out according to the Student's $t$ test at a statistical significance level of $p<0.01$.

\section{RESULTS}

In the presence of thymalin, the area of CD44 expression in passage 7 HSC significantly decreased by 2.76 times in comparison with control cultures (Table 1, Fig. 1). Thymalin did not affect the area of CD44 expression in passage $2 \mathrm{HSC}$ and the mean brightness of the expression of this molecule in cells of passages 2 and 7 (Tables 1, 2). CD44 molecule is expressed on HSC and regulatory $\mathrm{T}$ lymphocytes $[4,11]$. CD44 on HSC is a ligand for E- and L-selectins that maintain subpopulation of bone marrow stem cells $[2,9,11]$. CD44 is a receptor for hyaluronic acid [11] one of the main components of stem cell microenvironment. This differentiation cluster is involved in activation of proliferation, increasing survival rate of HSC, and modulating the effects of cytokines [11]. Thus, the decrease in the expression of CD44 in HSC under the action of thymalin can indicate its ability to stimulate cell differentiation, during which this molecule is no longer expressed. 

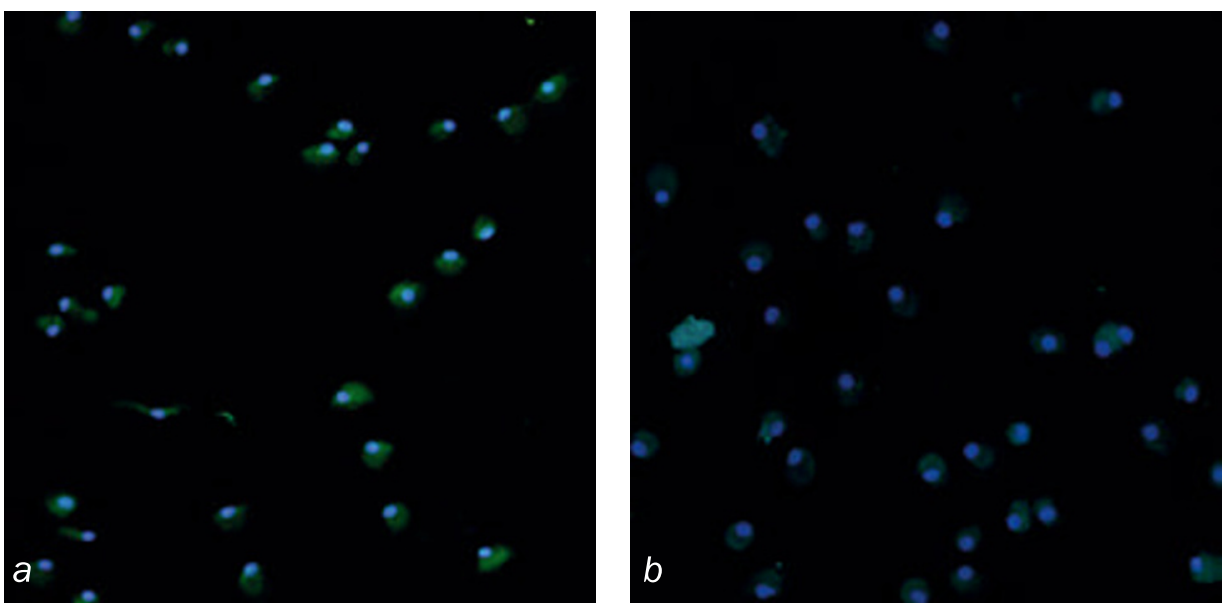

Fig. 1. CD44 expression in human hematopoietic stem cells, passage 7 . Immunofluorescence confocal microscopy, $\times 200$. Green fluorescence: expression of CD44 (Alexa Fluor 488). Blue fluorescence: cell nuclei were stained with DAPI. $a$ ) Control, $b$ ) thymalin.

The area of CD117 expression in passage 7 HSC under the influence of thymalin significantly decreased by 2.2 times in comparison with control cultures (Table 1, Fig. 2). Thymalin significantly increased the mean brightness of CD117 expression in passage 2 HSC by 2.16 times in comparison with the control (Table 2) and did not affect the area of CD117 expression in these cultures and the mean brightness of the expression of this molecule in passage 7 HSC (Tables 1, 2). Tyrosine kinase CD117 is a stem cell and neutrophil growth factor receptor. Activation of neutrophil growth factor receptor signaling pathway induces proliferation and early stages of HSC differentiation. Activation of CD117 receptor by neutrophil growth factor potentiates a number of cytokine-dependent signaling pathways, thereby promoting proliferation and differentiation of HSC $[3,12]$. The CD117 molecule is involved in the maintenance of HSC population in the blood and bone marrow. Expression of CD117 decreases during differentiation of immune cells, remaining only in mature neutrophils and some $\mathrm{CD} 8^{+} \mathrm{T}$ lymphocytes [3]. These findings suggest that thymalin in the course of short-term culturing stimulates the expression of CD117 on HSC, which can be considered as the initial stage of cell differentiation. During long-term cultivation, thymalin reduces the expression of CD117 on HSC. It can be hypothesized that this is related to activation of further HSC differentiation following the $\mathrm{CD} 117^{+}$cell stage under the effect of the thymus polypeptide complex.

The area of expression of CD28 in HSC of passages 2 and 7 under the influence of thymalin significantly increased by 2.98 and 6.93 times, respectively, in comparison with the control cultures (Table 1, Fig. 3). The mean brightness of CD28 expression in HSC of passages 2 and 7 under the influence of thymalin significantly increased by 2.03 and 2.05 times, respectively, in comparison with the control cultures (Table 2). Glycoprotein CD28 is expressed on thymocytes, $\mathrm{CD}^{+}, \mathrm{CD}^{+}$lymphocytes and is required for activation of mature T cells. The expression of CD28 is increased in antigen-presenting cells upon activation of cell Toll-like receptors, which leads to stimulation of cytokine synthesis. CD28 plays an important role in activation of immunity during viral diseases. In patients with a mild form of COVID-19, the number of $\mathrm{CD} 28^{+}, \mathrm{CD}^{+}$, and $\mathrm{CD} 8^{+}$lymphocytes increases, which indicates activation of the functions of the immune system. In a severe course of coronavirus infection, the number of $\mathrm{T}$ helpers and cytotoxic lymphocytes decreases with simultaneous decrease in the expression of CD28 glycoprotein on their surface [14]. It should

TABLE 1. Effect of Thymalin on the Relative Area of Expression of Signaling Molecules in the Culture of Human Hematopoietic Stem Cells $(\% ; M \pm S D)$

\begin{tabular}{l|c|c|c|c|c|c}
\hline \multirow{2}{*}{ Group } & \multicolumn{3}{|c|}{ Passage 2 } & \multicolumn{3}{c}{ Passage 7} \\
\cline { 2 - 7 } & CD44 & CD117 & CD28 & CD44 & CD117 & CD28 \\
\hline Control & $7.55 \pm 1.04$ & $15.25 \pm 1.68$ & $1.24 \pm 0.19$ & $5.47 \pm 0.45$ & $4.33 \pm 0.41$ & $2.01 \pm 0.16$ \\
Thymalin & $8.44 \pm 1.16$ & $13.23 \pm 1.71$ & $3.69 \pm 0.40^{*}$ & $1.98 \pm 0.13^{*}$ & $1.97 \pm 0.11^{*}$ & $14.56 \pm 1.75^{*}$ \\
\hline
\end{tabular}

Note. ${ }^{*} p<0.01$ in comparison with the control. 


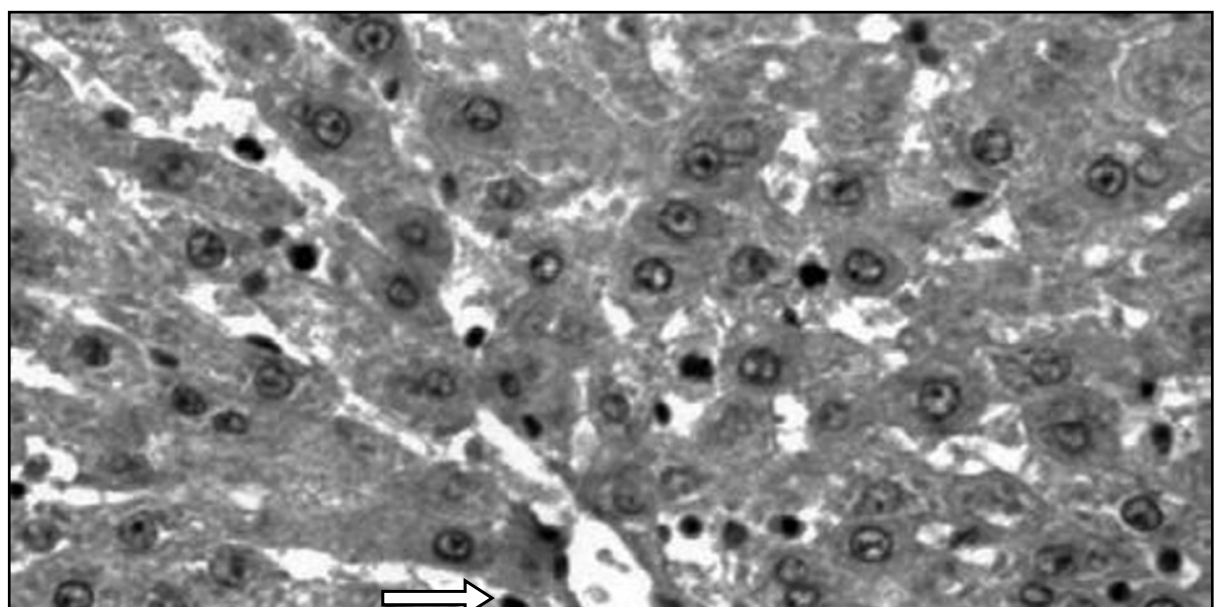

Fig. 2. CD117 expression in human hematopoietic stem cells, passage 7. Immunofluorescence confocal microscopy, $\times 200$. Red fluorescence: expression of CD117 (Alexa Fluor 647). Blue fluorescence: cell nuclei were stained with DAPI. $a$ ) Control, $b$ ) thymalin.

be noted that similar changes are induced by human immunodeficiency virus. It was found that CD28 expression on the membrane of $\mathrm{CD}^{+} \mathrm{T}$ lymphocytes increases their resistance to apoptosis and antitumor activity [8]. Stimulation of CD28 expression on HSC by thymalin can reflect activation of mature $\mathrm{T}$ cell differentiation and antiviral immunity.

It was found that during short-term culturing (up to passage 2), thymalin doubled the expression of the marker of early differentiation of immune cells CD117 on some HSC. At the same time, the number of HSC carrying CD117 glycoprotein on their surface decreased under the influence of thymalin. Thus, with a short-term effect on human stem cells, thymalin can step-by-step stimulate the initial stages of differentiation of these cells. CD $117^{+}$cells under the influence of thymalin proceed to a later stage of differentiation and lose their ability to synthesize CD117. This hypothesis is confirmed by the fact that the area and mean brightness of expression of the T lymphocyte marker CD28 in passage 2 HSC increase by 2-3 times under the action of thymalin.

During long-term culturing (up to passage 7), thymalin had even more pronounced effect on HSC differentiation towards $\mathrm{T}$ cells than during short-term culturing. Thymalin 2-3-fold reduced the expression of stem cell marker CD44 and molecule of the intermediate stage of HSC differentiation CD117 and almost 7-fold increased the synthesis of mature $\mathrm{T}$ cell marker CD28. Probably, the ability of thymalin to stimulate HSC differentiation was retained during long-term culturing. During short-term culturing, thymalin activated cell transition to the initial stage of differentiation, which was expressed in induction of the synthesis of glycoprotein CD117 on their surface. During long-term culturing in the presence of thymalin, $\mathrm{CD} 117^{+}$cells differentiated into mature $\mathrm{CD} 28^{+} \mathrm{T}$ lymphocytes. These data are consistent with the previously obtained results on the ability of di- and tripeptides to stimulate differentiation of immune cells in the bone marrow, thymus, embryonic liver, and peripheral blood of adults towards mature $\mathrm{T}$ helpers and cytotoxic $\mathrm{T}$ cells [5].

The obtained results are important for understanding of immunoprotective activity of thymalin in patients with acute respiratory diseases of viral etiology. In patients with viral infections, including those caused by SARS-CoV-2, activation of the functions of the immune system is observed at the initial stages of the disease, which manifests in an increase in the number of $\mathrm{CD} 28^{+}, \mathrm{CD}^{+}$, and $\mathrm{CD} 8^{+} \mathrm{T}$ lymphocytes in the blood [14]. If the course of COVID-19 proceeds according to an unfavorable scenario, for example, if

TABLE 2. Effect of Thymalin on the Mean Brightness of the Expression of Signaling Molecules in the Culture of Human Hematopoietic Stem Cells (arb. units; $M \pm S D$ )

\begin{tabular}{l|c|c|c|c|c|c}
\hline \multirow{2}{*}{ Group } & \multicolumn{3}{|c|}{ Passage 2 } & \multicolumn{3}{c}{ Passage 7} \\
\cline { 2 - 7 } & CD44 & CD117 & CD28 & CD44 & CD117 & CD28 \\
\hline Control & $22.15 \pm 2.85$ & $18.32 \pm 2.01$ & $16.49 \pm 1.70$ & $9.46 \pm 1.22$ & $21.89 \pm 1.14$ & $14.5 \pm 2.03$ \\
Thymalin & $24.78 \pm 2.34$ & $39.48 \pm 5.12^{*}$ & $33.48 \pm 4.03^{*}$ & $9.02 \pm 1.10$ & $22.88 \pm 1.12$ & $29.73 \pm 2.85^{\star}$ \\
\hline
\end{tabular}

Note. ${ }^{*} p<0.01$ in comparison with the control. 

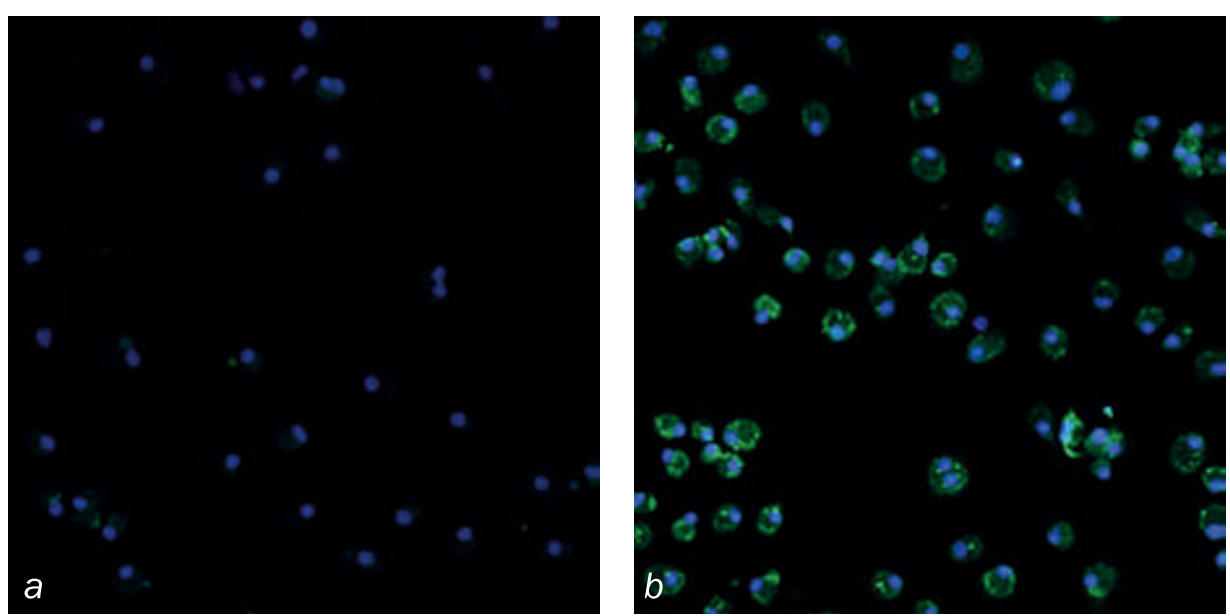

Fig. 3. CD28 expression in human hematopoietic stem cells, passage 7 . Immunofluorescence confocal microscopy, $\times 200$. Green fluorescence: expression of CD28 (Alexa Fluor 488). Blue fluorescence: cell nuclei were stained with DAPI. a) Control, $b$ ) thymalin.

the patient has concomitant chronic pathology, then the immune response fades, which correlates with a decrease in the number of blood $\mathrm{T}$ lymphocytes and can lead to death [10].

It is possible that the antiviral effect of thymalin is expressed in compensatory stimulation of HSC differentiation into $\mathrm{CD} 28^{+} \mathrm{T}$ lymphocytes at the stage of immunity suppression in an unfavorable course of viral infection. Immunomodulators are one of the possible ways of prevention and complex therapy of mild forms of COVID-19 [13]. Thus, thymalin can be considered as a peptide preparation for the prevention of viral infections, including infection caused by SARS-CoV-2.

\section{REFERENCES}

1. Anisimov VN, Khavinson VKh. Peptide bioregulation of aging: results and prospects. Biogerontology. 2010;11(2):139149.

2. Chen C, Zhao S, Karnad A, Freeman JW. The biology and role of CD44 in cancer progression: therapeutic implications. J. Hematol. Oncol. 2018;11(1):64. doi: 10.1186/s13045-0180605-5

3. Frumento G, Zuo J, Verma K, Croft W, Ramagiri P, Chen FE, Moss P. CD117 (c-Kit) is expressed during CD8+ T cell priming and stratifies sensitivity to apoptosis according to strength of TCR engagement. Front. Immunol. 2019;10:468. doi: 10.3389/fimmu.2019.00468

4. Godoy GJ, Paira DA, Olivera C, Breser ML, Sanchez LR, Motrich RD, Rivero VE. Differences in T regulatory cells between mouse strains frequently used in immunological research: Treg cell quantities and subpopulations in NOD, B6 and BALB/c mice. Immunol. Lett. 2020;223:17-25.

5. Khavinson V, Linkova N, Diatlova A, Trofimova S. Peptide regulation of cell differentiation. Stem Cell Rev. Rep. 2020;16(1):118-125.

6. Khavinson VKh. Peptides and ageing. Neuroendocrinol. Lett. 2002;23(Suppl. 3):11-144.
7. Khlystova ZS, Kalinina II, Shmeleva SP. Thymalin in developing respiratory organs of human fetus. Bull. Exp. Biol. Med. 2003;135(6):600-602.

8. Kim KH, Kim HK, Kim HD, Kim CG, Lee H, Han JW, Choi SJ, Jeong S, Jeon M, Kim H, Koh J, Ku BM, Park SH, Ahn MJ, Shin EC. PD-1 blockade-unresponsive human tumorinfiltrating CD8+ T cells are marked by loss of CD28 expression and rescued by IL-15. Cell Mol. Immunol. 2020. doi: 10.1038/s41423-020-0427-6

9. Li JP, Hsieh MJ, Chou YE, Chao YH, Tsao TC, Yang SF. CD44 gene polymorphisms as a risk factor for susceptibility and their effect on the clinicopathological characteristics of lung adenocarcinoma in male patients. Int. J. Environ. Res. Public Health. 2020;17(9):2981. doi:10.3390/ijerph17092981

10. Mehta P, McAuley DF, Brown M, Sanchez E, Tattersall RS, Manson JJ; HLH Across Speciality Collaboration, UK. COVID-19: consider cytokine storm syndromes and immunosuppression. Lancet. 2020;395:1033-1034.

11. Morath I, Hartmann TN, Orian-Rousseau V. CD44: more than a mere stem cell marker. Int. J. Biochem. Cell Biol. 2016;81(Pt A):166-173.

12. Myburgh R, Kiefer JD, Russkamp NF, Magnani CF, Nuñez N, Simonis A, Pfister S, Wilk CM, McHugh D, Friemel J, Müller AM, Becher B, Münz C, van den Broek M, Neri D, Manz MG. Anti-human CD117 CAR T-cells efficiently eliminate healthy and malignant CD117-expressing hematopoietic cells. Leukemia. 2020. doi: 10.1038/s41375-020-0818-9

13. Scavone C, Brusco S, Bertini M, Sportiello L, Rafaniello C, Zoccoli A, Berrino L, Racagni G, Rossi F, Capuano A. Current pharmacological treatments for COVID-19: what's next? Br. J. Pharmacol. 2020. doi:10.1111/bph.15072

14. Wang F, Hou H, Luo Y, Tang G, Wu S, Huang M, Liu W, Zhu Y, Lin Q, Mao L, Fang M, Zhang H, Sun Z. The laboratory tests and host immunity of COVID-19 patients with different severity of illness. JCI Insight. 2020;5(10):e137799. doi: 10.1172/jci.insight. 137799

15. Zhukova GV, Schikhlyarova AI, Barteneva TA, Shevchenko AN, Zakharyuta FM. Effect of Thymalin on the tumor and thymus under conditions of activation therapy in vivo. Bull. Exp. Biol. Med. 2018;165(1):80-83. 